\title{
Influence of non-uniform deformation on photoelectric properties of crystalline silicon
}

\author{
O.V. Vakulenko, S.V. Kondratenko \\ Taras Shevchenko Kyiv Univ., 6 Glushkova Prosp., 252127 Kyiv, Ukraine \\ E-mail:kondr@hq.ups.kiev.ua.
}

\begin{abstract}
Measurements of the photoconductivity (PC) and photomagnetic effect (PME) spectra of crystalline silicon were carried out for the sample under the non-uniform bend deformation. This deformation causes a decrease of the photoconductivity spectrum drop in the short-wave region when illuminating the stretched surface. Under constant deformation conditions the PME spectrum form is changed only in the long-wave region. Obtained data are explained by diffusion length decreasing as a consequence of decreasing diffusion coefficient under the influence of a strain gradient.
\end{abstract}

Keywords: photoconductivity, photomagnetic effect, bend deformation, crystalline silicon

Paper received 04.08.00, revised manuscript received 12.09.00, accepted for publication 12.12.00.

\section{Introduction}

The processes of defects creating in the crystalline silicon have been the subject of many theoretical and experimental investigations. It has been shown that the $\mathrm{Si}-$ $\mathrm{SiO}_{2}$ interface contains the transition region $-\mathrm{a} \mathrm{SiO}_{x}$ $(x<2)$ layer with disrupted stoichiometry. This layer is oversaturated with different kinds of defects and has interior strains because of distinctions in thermal expansion coefficients between oxide and silicon substrate [1,2]. Elastic interaction between the point defects and non uniform strain fields results in migration of defects in silicon [3]. Therefore, the creation of the mechanical strain gradient probably is responsible for changing the electronic properties of the silicon surface, which caused by creation of a mobile defect concentration gradient.

On the other hand, it has been known, that the mechanical strain in the silicon substrate has an essentially impact on the formation of the porous silicon produced by the anodization of the crystalline silicon. Namely, in the papers $[4,5]$ it has been shown that the creation of mechanical strains in a crystalline silicon substrate before the process of anodization yields in of stimulation of porous silicon layer growth. Such result in the paper [5] is explained by changing a surface electrochemical potential under mechanical strains, which causes changes in the value of the HF - Si barrier and therefore in the thickness of the porous silicon layer.
Presented results create a base to assume the possibility of changing the photoelectric properties of crystalline silicon under non-uniform mechanical strains. The aim of our work was to investigate changes in the PC and PME spectra of crystalline silicon under bend deformation.

Such investigations serve as the modelling ones when studying photoelectric properties of porous silicon. As it has been known that porous silicon has a lot of non-uniform strains and is characterized by high concentration of defects as compared to crystalline silicon. Nevertheless, porous silicon has rather high photosensitivity [6].

\section{Experiment}

The crystalline $n$-type silicon samples with resistivities $200 \Omega \cdot \mathrm{cm}$ were used for measurements. Mechanical strains were created by the scheme of three-point bend (Fig.1a). The samples have a parallelepiped form with dimensions $2 \times 3 \times 8 \mathrm{~mm}^{3}$. The spectra PC and PME were measured for deformed and non-deformed samples. The value of load was $\sim 5 \mathrm{kgf} \cdot \mathrm{mm}^{-2}$. Stretched and pressed regions simultaneously exist under such bend deformation. Stretched surface was illuminated in our case.

The samples were illuminated normally to the surface from the side where contacts are disposed. At the same time, the contacts were not illuminated. Measurements were carried out on the standard spectrometer IKS- 12 . The $70 \mathrm{~W}$ halogen lamp served as a source of light. The 
measured spectral responses for PC and PME were brought to the constant number of the light quantum of exciting irradiation.

\section{Results and discussion}

In the Fig. 1 the PC spectra are shown for the bend deformed (curve 1) and non-deformed (curve 2) sample of the crystalline silicon. The practically same shape of the measured PC spectra in the long-wave region points to the unchanged energy gap on the such value of the mechanical load.

The decrease of the PC spectrum drop in the shortwave region is observed for the deformed sample as compared to the PC spectrum for the non-deformed sample. Obtained results have a few explanations. First, these can be explained by decreasing of the influence of surface recombination. According to the model, proposed in the paper [7], the surface recombination rate depends on the surface potential, because the change of the surface recombination rate may be explained by the change of the surface potential under the mechanical deformation [6]. The PC spectrum shape (Fig.1), namely, the considerable drop in the short-wave region, is an evidence of significant value of a surface recombination rate for our samples $\left(\sim 10^{4} \mathrm{~cm} / \mathrm{s}\right)$ [8]. Bearing in the mind this high value, the change of the PC spectrum shape under deformation may be explained by decrease of the surface recombination rate by several times with persistence of other parameters. Secondly, the change of the PC spectrum shape may take place in consequence of the change of lifetime and/or diffusion coefficient under the constant surface recombination rate. Because, at the great surface recombination rate the rate of losses of the charge carriers will be confined not by «passing» capability of the surface recombination centers but diffusion of the nonequilibrium charge carriers to the surface.

The PME spectra have been measured at the same con-ditions that the PC spectra measurements to clear

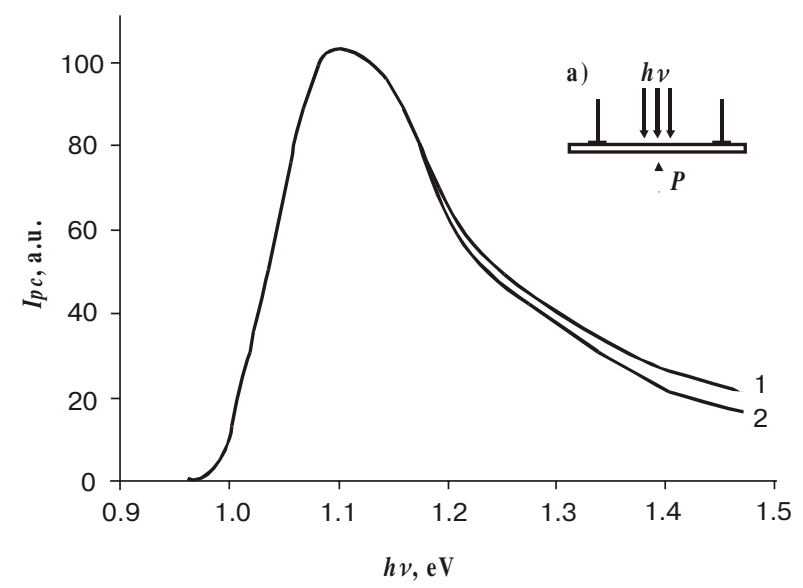

Fig. 1. The PC spectrum for the bend deformed (1) and nondeformed (2) sample. the uncertainty at the explanation of the differences of PC spectrum shape. The PME spectrum without deformation is shown in Fig. 2, and in Fig. 3-under the bend deformation. It turned out that the PME spectra were abnormal, that is their sign was opposite to the normal one. That indicates presence of a spatial charge layer depleated by the majority carriers and the great rate of surface recombination [9]. The sign distinction between normal and abnormal PME was verified by means of additional illumination in the region of the surface absorption that, as it is well known, results in changing value of surface potential. Under these conditions, the change of the PME sign by the opposite one was observed in the long-wave region. The power of the $100 \mathrm{~W}$ halogen lamp was not enough for changing the sign of the PME spectrum in all spectral range.

The model given in the paper [9] used for describing the abnormal PME spectra. According to this model the short circuit current is determined from following equations:

$$
\begin{aligned}
& I_{\mathrm{PME}}=q\left(\mu_{n}+\mu_{p}\right) \frac{H}{c} I w Z \\
& Z=-\left(\frac{1-e^{-\alpha w}}{\alpha w}-\frac{e^{-\alpha w}}{1+\alpha L}\right),
\end{aligned}
$$

where $w$ is the thickness of the spatial charge layers; $\alpha-$ the absorption coefficient; $L$ - the diffusion length.

The curves of the relative PME spectral distribution calculated using formula (2) are shown by a solid line in the Fig.2-3. The parameters $w$ and $L$ were calculated under conditions of the maximum correlation coefficient between experimental and calculated data. So, the spatial charge length and diffusion length are $w=20 \mu \mathrm{m}$ and $L=98 \mu \mathrm{m}$, respectively, for the non-deformed sample. The creation of the bend deformation leads to decreasing of the diffusion length $(L=53 \mu \mathrm{m})$, and, at the same time, the thickness of the spatial charge layer is not changed.

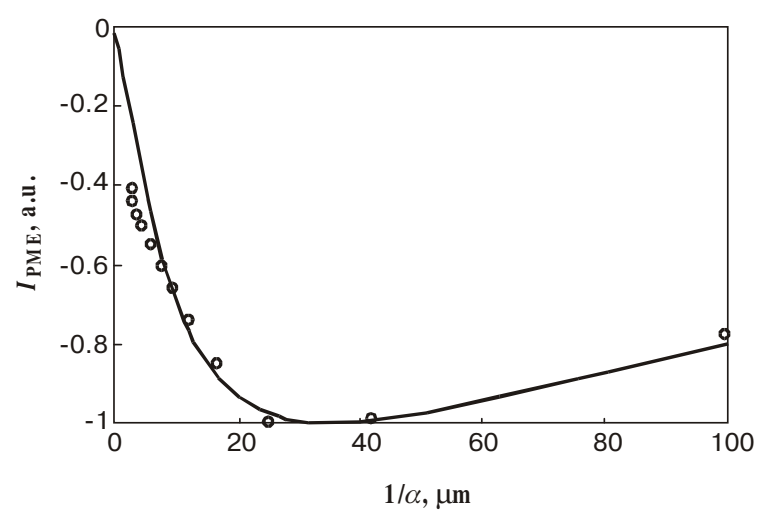

Fig. 2. The spectral response of the PME for the deformed sample and the result of calculation (solid line) with the parameters: $L=98 \mu \mathrm{m}, w=20 \mu \mathrm{m}$.

SQO, 3(4), 2000 
O.V. Vakulenko, S.V. Kondratenko: Influence of non-uniform deformation on the ...

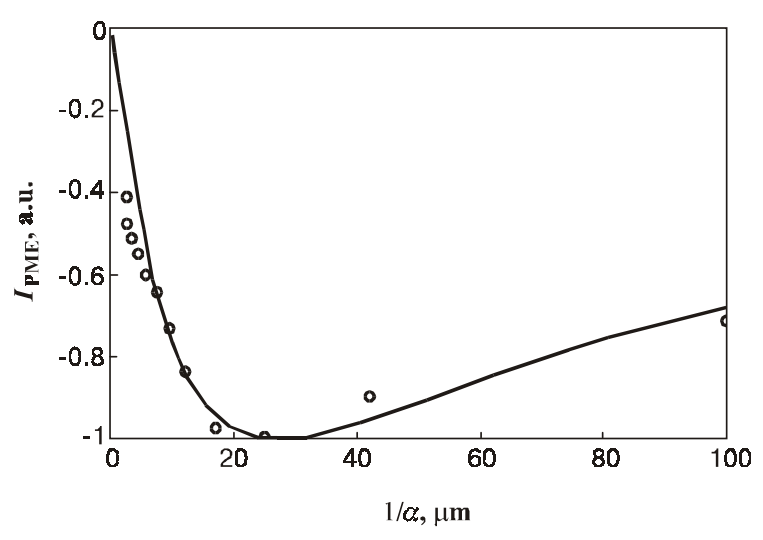

Fig. 3. The spectral response of the PME for the deformed sample and the result of calculation (solid line) with the parameters: $L=53 \mu \mathrm{m}, w=20 \mu \mathrm{m}$.

The obtained data may be explained in the following way. The equal values of the short circuit currents in the short-wave region is evidence of that the value of the surface recombination rate is constant. Because the change of this value in a several time, that following from the analysis of the PC spectrum shape, might lead to considerable changing the PME form in the short-wave region even to the change of PME sign, that is not observed experimentally. Besides, the absence of changing the thickness of the spatial charge layer indicates the invariability of the surface potential value under the deformation. Indeed, the concentration of the majority and minority charge carriers in the case of depletion layers is small, and the key role in the compensation of the surface charge is played by the stationary charge of the noncompensated donors or acceptors [7]. Obviously, the variation of the surface potential with distance from the surface in this case is relatively slow, while the thickness of the spatial charge layer is relatively large and the significant change of the value of the spatial charge by means of mechanical deformation is not possible.

In this case the bend deformation leads to the diffusion length decreasing for the stretched surface. It appears as the PME spectrum changes in the short-wave region and decreasing surface recombination influence ascertained in studies of PC spectra. The reason of the diffusion length decreasing may be the changes both the diffusion coefficient and the lifetime of the charge carriers. But, the lifetime changing of the charge carriers under deformation might lead to changing concentration of the equilibrium charge carriers, and, therefore, to changing the spatial charge thickness (screening length), that has not yet been observed experimentally. Therefore, the reason of changing the PC and PME spectrum shape in our case is the decreasing value of the diffusion coefficient.

We gratefully acknowledge B. K. Serdega for helpful discussions.

\section{References}

1. V.G. Litovchenko, A.P. Gorban Osnovy phiziky mikroelectronnikh sistem metal - dielectric - poluprovodnik // (The phy-sics base of the microelectronic systems metal - dielectric - semiconductor), Kiev: Nauk. Dumka, 1978, p. 263 (in Russian).

2. N.D.Gerasimenko, V.N.Mordkovich, Radiacionnie effektu v sisteme poluprovodnik - dielektrik // Poverchnost, No.6 pp.5-19 (1987) (in Russian).

3. E.N.Poindexter et. al. Electronic traps and $P_{b}$ centers at the $\mathrm{Si} / \mathrm{Sio}_{2}$ interface: Band-gap energy distribution // J.Appl.Phys. 56(5), pp. 2844-2849 (1984).

4. V.A. Makara, M.S. Boltovets, O.V. Vakulenko, O.I. Datsenko, V.I. Kryvohyzhyna, O.V. Rudenko, Effect of substrate defects on the luminescent properties of porous silicon layers In Frontiers in Nanoscale Science of Micron / Submicron Devices, Nato ASI Series 328, 1996, p. 409.

5. G.K. Moroz, A.V. Zhersdev. K voprosu o mehanizme formirovania poristogo kremniya // FTP. 28(6), pp.949-953 (1994) (in Russian).

6. O.V. Vaculenko, S.V. Kondratenko, V.A. Makara, O.V. Rudenko, B.M.Shutov. Fotoelectronni vlastivosti poruvatogo kremniu // Ukr.Fiz.Zhurn. 44(12), pp.1465-1467 (1999) (in Ukrainian).

7. A.V.Rzhanov // Electronye processy na poverchnosty poluprovodnikov (The electronic processes on the semiconductor surface). Nauka, Moscow, 1971. p. 480 (in Russian).

8. V.K. Subashiev, V.A. Petrusevich, G.B. Dubrovsky, Opredelenie recombinacionnih postoyannih iz krivoy spectralnogo raspredelenia fotoprovodimosty // FTT, 11(5), pp.1022-1024 (1960) (in Russian).

9. S.M.Gorodetsky, N.S.Zhdanovich, U.I.Ravich. Anomalniy fotomagnitniy effect v kremnii // FTP, 7(7), pp.1270-1279 (1963) (in Russian). 